\title{
Involvement of regucalcin gene promoter region-related protein-p117, a transcription factor, in human obesity (Review)
}

\author{
MASAYOSHI YAMAGUCHI $^{1}$ and TOMIYASU MURATA ${ }^{2}$ \\ ${ }^{1}$ Department of Hematology and Medical Oncology, Winship Cancer Institute, Emory University School of Medicine, \\ Atlanta, GA 30322, USA; ${ }^{2}$ Laboratory of Analytical Neurobiology, Faculty of Pharmacy, \\ Meijo University, Nagoya, Aichi 468-8503, Japan
}

Received December 16, 2016; Accepted February 15, 2017

DOI: $10.3892 /$ br.2017.874

\begin{abstract}
Regucalcin gene promoter region-related protein-p117 (RGPR-p117; gene symbol, rgpr-117) was identified in 2001 as a novel transcription factor that specifically binds to a nuclear factor I consensus motif, TTGGC(N) ${ }_{6} \mathrm{CC}$ in the promoter region of the regucalcin (rgn) gene. The human RGPR-p117 gene consists of 26 exons spanning $4.1 \mathrm{kbp}$ and is localized on chromosome 1q25.2. The nuclear translocation of cytoplasm RGPR-p117 is mediated via the protein kinase C-dependent signaling pathway. Overexpression of RGPR-p117 enhances the transcription activity of rgn, and a protective effect on cell death by inhibition of gene expression levels of caspase-3, caspase- 8 and FADD proteins that possess the TTGGC motif in the promoter region of those genes was revealed. RGPR-p117 has a crucial role as a transcription factor. Notably, RGPR-p117 was shown to localize in the plasma membranes, mitochondria and microsomes (endoplasmic reticulum; ER). RGPR-p117, which is located in the ER, was also shown to have a role as an ER export factor implicated in the transports of proteins and lipids. As a result of this finding, it was proposed in 2007 that RGPR-p117 is renamed SEC 16 homolog $\mathrm{B}$, endoplasmic reticulum export factor (SEC16B). Recently, there is increasing evidence that RGPR-p117/SEC16B may be involved in human obesity. Thus, the current review presents data regarding the involvement of RGPR-p117 in human obesity.
\end{abstract}

\section{Contents}

1. Introduction

Correspondence to: Dr Masayoshi Yamaguchi, Department of Hematology and Medical Oncology, Winship Cancer Institute, Emory University School of Medicine, C-5054, 1365 C Clifton Road, NE, Atlanta, GA 30322, USA

E-mail: yamamasa1155@yahoo.co.jp

Key words: regucalcin gene promoter region-related protein-p117, SEC16 homolog B, endoplasmic reticulum export factor, regucalcin, transcription factor, nuclear localization, apoptosis, endoplasmic reticulum export factor, obesity
2. Pivotal role of RGPR-p117 as a transcription factor

3. Role of RGPR-p117/SEC16B as an endoplasmic reticulum export factor

4. Involvement of RGPR-p117/SEC16B in human obesity

5. Conclusion

\section{Introduction}

Regucalcin gene promoter region-related protein-p117 (RGPR-p117; gene symbol, rgpr-p117) was originally discovered as a novel transcription factor for regucalcin (rgn) gene expression in $2001(1,2)$. The RGPR-p117 gene consists of 26 exons spanning $\sim 4.1 \mathrm{kbp}$ and is localized on human chromosome 1q25.2 (2). RGPR-p117 conserves a leucine zipper motif (2). Thus, RGPR-p117 was termed as the leucine zipper transcription regulator 2 (Lztr2). Phosphorylated RGPR-p117 in the nucleus specifically binds to a nuclear factor I consensus motif, TTGGC(N) ${ }_{6} \mathrm{CC}$ that is located on the $5^{\prime}$-flanking region of the regucalcin (3-8). Regucalcin has been shown to be pivotal as a suppressor in various cell signaling pathways and in transcription activity for maintaining cell homeostasis and disorder in various cell and tissue types (9-11).

In 2007 it was proposed that RGPR-p117 be renamed to SEC16 homolog B, endoplasmic reticulum export factor (SEC16B) (12). RGPR-p117 has a putative role as an endoplasmic reticulum (ER) export factor implicated in the transports of proteins and lipids in the ER (13). In recent years, growing evidence indicates that the RGPR-p117/SEC16B gene is involved in human obesity and type 2 diabetes (14). However, to the best of our knowledge, the role of RGPR-p117/SEC16B as an ER export factor implicated in lipid metabolism has not been investigated. Thus, the aim of the current review was to discuss the findings regarding the role of RGPR-p117/SEC16B as an ER export factor implicated in lipid metabolism and its involvement in human obesity and type 2 diabetes.

\section{Pivotal role of RGPR-p117 as a transcription factor}

RGPR-p117 enhances rgn gene expression. The RGPR-p117 gene, rgpr-p117, is expressed in various species, including 
humans, rats, mice, dogs, cows, pigs, rabbits, chickens, frogs (Xenopus), fish (Zebrafish), C. elegans and yeast $(8,15-17)$. The human rgpr-pl17 is located in human chromosome $1 \mathrm{q} 25.2$ and is organized with a minimum of 26 exons spanning $\sim 4.1 \mathrm{kbp}(2,8)$. RGPR-p117 in the human liver consists of 1,060 amino acid residues with a calculated molecular mass of $117 \mathrm{kDa}$ and an estimated isoelectric point $(\mathrm{p} I)$ of $5.71(2,15)$. RGPR-p117 is highly homologous in various types of mammalian species, indicating a functional significance in vertebrate species (15-17). Mammalian RGPR-p117 was confirmed to highly conserve a leucine zipper motif, which is a characteristic structure of nuclear transcription factors $(2,17-21)$. The leucine zipper pattern is conserved in numerous gene regulatory proteins, such as CCATT-box/enhancer binding protein (22), nuclear oncogenes, fos and jun (23), cAMP response element (CRE) binding proteins (CREB, CRE-BP1 and ATFs) (24), c-Myc, L-Myc, and N-Myc oncogenes (25), and octamer-binding transcription factor 2 (Oct-2/OTF-2) (26). This finding may support the hypothesis that RGPR-p117 is a transcription factor.

RGPR-p117 was demonstrated to enhance the rgn promoter activity $(7,8)$. RGPR-p117 possesses various motifs associated with post-translational modification, such as casein kinase II phosphorylation, protein kinase C (PKC) phosphorylation, $\mathrm{N}$-myristoylation, $\mathrm{N}$-glycosylation and amidation (2). Modified native RGPR-p117 may bind to the TTGGC motif in the promoter region of the rgn in the nucleus $(5,8)$. A computer analysis of subcellular localization of RGPR-p117 from six vertebrates demonstrated a higher probability of nuclear localization, particularly in rats and mice $(78.3 \%)(2,17)$. RGPR-p117 was demonstrated to localize in the cytoplasm and nucleus of modeled, normal rat kidney proximal tubular epithelial NRK-52E cells with immunocytochemical analysis using transient HA-RGPR-p117/phCMV2-transfected cells (5). This nuclear localization was enhanced by a culture with fetal bovine serum, parathyroid hormone or phorbol 12-myristate 13 -acetate which activates various protein kinases, including PKC $(5,27-29)$.

Overexpression of RGPR-p117 was found to enhance regucalcin mRNA and protein expression levels in NRK52E cells (6). Notably, the stimulatory effects of RGPR-p117 overexpression on the rgn promoter activity in NRK52E cells transfected with the $-710 /+18$ LUC construct (which contains the TTGGC sequence) disappeared in the cells that were transfected with the LUC construct with the deleted TTGGC motif (7).Furthermore, the stimulatory effects of overexpressed RGPR-p117 on regucalcin mRNA expression were blocked by culture with staurosporine, an inhibitor of PKC (30), while this stimulatory effect was not suppressed in the presence of dibucaine, an inhibitor of $\mathrm{Ca}^{2+} /$ calmodulin-dependent protein kinase (29) or PD98059, an inhibitor of MAP kinase (31). The stimulatory effects of RGPR-p117 on regucalcin mRNA expression were mediated through PKC-dependent signaling process that stimulates protein phosphorylation in the nucleus of NRK52E cells (6). Furthermore, regucalcin mRNA expression has been shown to be stimulated through $\mathrm{Ca}^{2+}$ signaling factors, which have been associated with PKC in modeled rat hepatoma H4-II-E cells (29) and NRK52E cells (32). In addition, stimulatory effects of overexpressed RGPR-p117 on the rgn gene promoter activity were clearly blocked by culture with vanadate, an inhibitor of tyrosine kinase $(6,33)$. Such an effect was not revealed in the case of okadaic acid, an inhibitor of protein serine/threonine phospharase $(6,33)$. The inhibition of protein tyrosine phosphatases contributed to demonstrating the stimulatory effects of RGPR-p117 on the rgn promoter activity $(6,33)$.

As described above, phosphorylated RGPR-p117 in the nucleus may directly bind to the TTGGC motif of the rgn promoter region and enhance the transcription activity of $r g n$ expression $(5,7,8)$.

RGPR-p117 regulates the gene expression implicated in apoptotic cell death. Overexpression of RGPR-p117 did not exhibit a significant effect on proliferation in NRK52E cells (34). Notably, overexpressed RGPR-p117 demonstrated protective effects on apoptotic cell death induced by tumor necrosis factor- $\alpha$, lipopolysaccharides or Bay K8644 in NRK-52E cells (34). These factor-induced cell deaths were clearly prevented by culture with inhibitor of caspase-3, which activates endonuclease to mediate nuclear DNA cleavage into oligonucleosome fragments $(35,36)$ in NRK52E cells (37). Mechanically, overexpression of RGPR-p117 was demonstrated to decrease the mRNA expression levels of Fas-associating death domain (FADD) protein, caspase-8, caspase-9, and caspase- 3 that are implicated in the stimulation of apoptotic cell death in NRK52E cells (37). RGPR-p117 may exert protective effects on apoptotic cell death due to suppressing the gene expression of various proteins implicated in the stimulation of apoptosis (37). Notably, the TTGGC motif, which is present in the $r g n$ promoter region $(1,2)$, is also located in the promoter region of the genes of caspase- 3 , caspase- 8 or FADD $(2,37)$. RGPR-p117, which binds to the TTGGC motif in the promoter region of caspase-3, caspase- 8 or FADD, may repress the gene expression levels of caspase-3, caspase- 8 or FADD in the nucleus of NRK52E cells.

Thus, RGPR-p117 may be critical in cell regulation as a transcription factor in the gene expression of various proteins. As summarized in Fig. 1, cytoplasm RGPR-p117 is translocated to the nucleus via a signaling mechanism associated with $\mathrm{Ca}^{2+}$-dependent PKC in cells. Nuclear RGPR-p117, which is phosphorylated by various protein kinases, including $\mathrm{PKC}$, specifically binds to the TTGGC motif in the promoter region of the various target genes in the nucleus, leading to regulation of the gene expression in cells. RGPR-p117 possesses a leucine zipper motif, which is conserved in various species $(1,2)$. Thus, RGPR-p117 may be crucial role as a transcription factor for gene expression in various cell and tissue types.

\section{Role of RGPR-p117/SEC16B as an ER export factor}

The organelle distribution of RGPR-p117 has been analyzed in NRK-52E cells transfected with HA-RGPR-p117/phCMV2(34). Results of western blot analysis for HA-RGPR-p117 demonstrated that this protein localizes in the plasma membranes, cytoplasm, mitochondria, ER and nucleus of the cells (34). RGPR-p117 may have a regulatory role in these organelles. Of note, overexpression of RGPR-p117 was shown to decrease the protein and DNA contents in NRK52E cells (34).

RGPR-p117 was shown to possess higher homology to SEC16B $(2,12)$. Furthermore, SEC16B has been reported to 


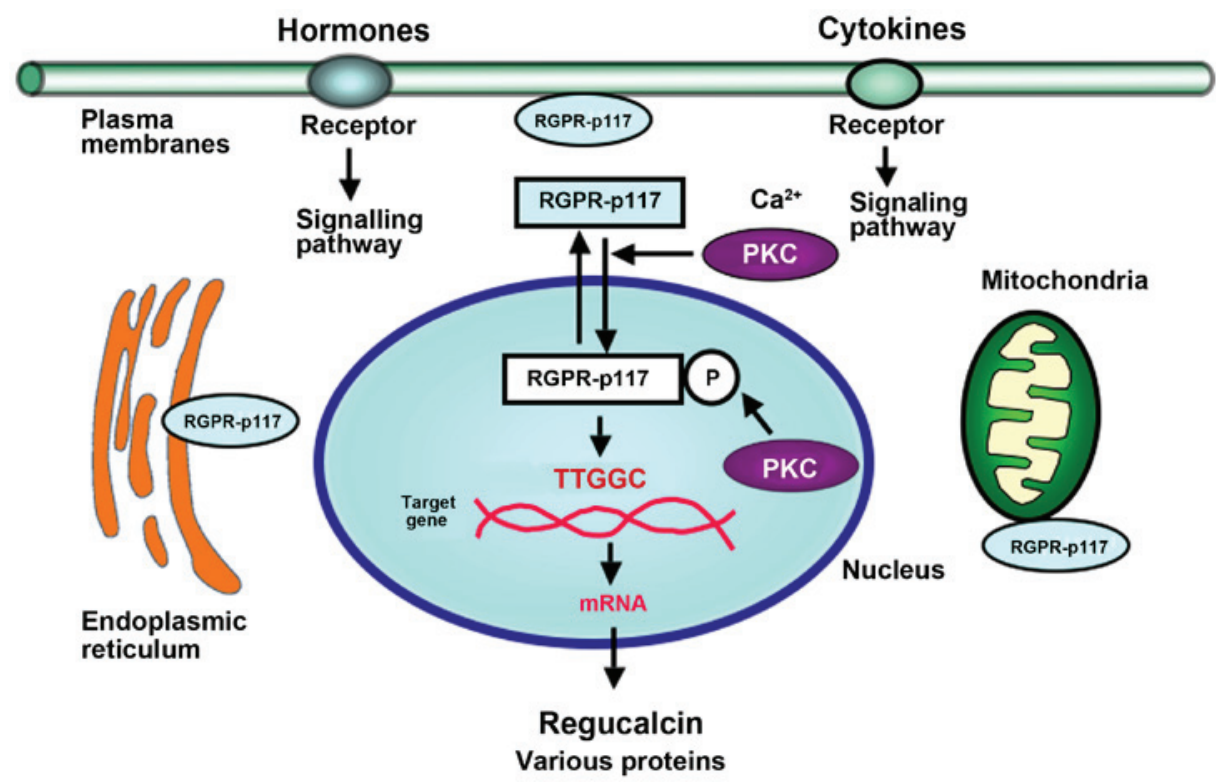

Figure 1. RGPR-p117 has a crucial role as a transcription factor. Cytoplasmic RGPR-p117 is translocated to the nucleus, which is enhanced via activation of $\mathrm{Ca}^{2+}$ signaling-dependent PKC. Nuclear RGPR-p117 is phosphorylated by PKC. Phosphorylated RGPR-p117 in the nucleus binds to the TTGGC motif in the promoter region of the regucalcin gene, as well as other genes, and it regulates the transcription activity of target genes. RGPR-p117 exerts suppressive effects on DNA and protein synthesis, and protective effects on apoptotic cell death. Furthermore, RGPR-p117 is localized in the plasma membranes, mitochondria and ER in cells. RGPR-p117 may have a role as an ER export factor for lipids that are implicated in obesity. RGPR-p117, regucalcin gene promoter region-related protein; PKC, protein kinase C; ER, endoplasmic reticulum; P, phosphorylated.

be an ER export factor (13). RGPR-p117 was demonstrated to bind to the ER of normal rat kidney NRK52E cells (35). RGPR-p117 has been suggested to perform a functional role in ER export within cells.

The ER represents the entry point into the secretory pathway, and from here newly synthesized proteins and lipids are delivered to the Golgi $(13,38,39)$. The selective cargo export from the ER is mediated by coat protein II (COPII)-assembly at specific sites of the ER, the transitional ER (tER) $(13,38)$. The peripheral membrane protein, SEC16 localizes to tER and may be key to the organization of these sites, and this protein defines the tER and is hypothesized to act as a scaffold for COPII-assembly $(13,38)$. SEC16 may be crucial in the formation of COPII vesicles, which mediate transport of proteins and lipids from the ER to the Golgi apparatus $(13,38)$. Two isoforms of SEC16 have been reported as follows: The larger isoform, SEC16A, and the smaller isoform, SEC16B $(13,38)$. However, the functional differences between the two isoforms are ill-defined $(13,38)$. SEC16B was shown as not functionally redundant in ER export and transitional ER organization $(13,38)$. Characterization of the localization and dynamics of SEC16B relative to Sec16A provide evidence that SEC16B may be a minor, or perhaps specialized, form of SEC16 and that it is not functionally redundant with SEC16A (38-40).

SEC16B, as well as SEC16A, defines ER exit sites, where COPII vesicles are formed in mammalian cells $(38,39)$. SEC16A and SEC16B, mammalian orthologs of yeast SEC16, are scaffold proteins that organize ER exit sites by interacting with COPII components (39). Overexpressed SEC16B targeted the entire ER, whereas SEC16A was predominantly cytosolic (39). Concomitant with the overexpression of SEC16B, peroxisomal biogenesis factor 3 (PEX3) and PEX16 were redistributed from peroxisomes to SEC16B-positive ER membranes (39).
Knockdown of SEC16B, but not SEC16A, by RNAi affected the peroxisome morphology, inhibited the transport of PEX16 from the ER to the peroxisomes, and suppressed the expression of PEX3 (39). These phenotypes were reversed by the expression of RNAi-resistant SEC16B (39). SEC16B, but not SEC16A, was demonstrated to regulate the transport of peroxisomal biogenesis factors from the ER to peroxisomes in mammalian cells (39). Furthermore, the C-terminal region of SEC16B, which is not conserved in SEC16A, is required for this function $(38,39)$. SEC16B, in ER areas other than ER exit sites, was demonstrated to perform this role, providing an explanation of how secretory and peroxisomal trafficking from the ER are distinguished (38).

As described above, RGPR-p117 may be involved in ER export process in the cells. However, more studies are required to further elucidate this process.

\section{Involvement of RGPR-p117/SEC16B in human obesity}

There is growing evidence to indicate that RGPR-p117/SEC16B may be involved in human obesity (41-46). Obesity is a major health issue worldwide, with increasing prevalence in adults and children from developed and developing countries (41). Obesity causes various chronic diseases, including cardiovascular and respiratory disease, osteoarthritis, hypertension, stroke, type II diabetes, obstructive sleep apnea, and different types of cancer (41). At least 52 genetic loci have been shown to associate with obesity-associated traits (42). Genome-wide association studies (GWAS) effectively reveal an association between single nucleotide polymorphisms and different measures of obesity, and have identified a number of genes associated with human body weight (41-46). Previous GWAS have identified numerous genes of proteins associated with obesity, including leptin 
(LEP), LEP receptor, proopiomelanocortin, proprotein convertase subtilisin/kexin type 1, $\alpha$-ketoglutarate dependent dioxygenase (FTO), melanocortin 3 receptor (MC3R), MC4R, glucosamine-6-phosphate deaminase 2 (GNPDA2), transmembrane protein 18 , glutaminyl-peptide cyclotransferase like/gastric inhibitory polypeptide receptor, brain derived neurotrophic factor (BDNF), ETS variant 5 (ETV5), mitogen-activated protein kinase kinase 5/SKI family transcriptional corepressor 1, SEC16B, single-minded family bHLH transcription factor 1, and tankyrase/methionine sulfoxide reductase A (45). RGPR-p117/SEC16B may be a kind of gene implicated in human obesity.

The involvement of SEC16B in obesity has been investigated in model animals using obese diabetic rats (47). Schmid et al (47) performed an expression analysis of 14 genes, namely BDNF, ETV5, Fas apoptotic inhibitory molecule 2 (FAIM2), FTO, GNPDA2, potassium channel tetramerization domain containing 15, lysophospholipase like 1 (LYPLAL1), MC4R, mitochondrial carrier 2, neuronal growth regulator 1 , neurexin 3 , transmembrane protein 18 , SEC16B, and transcription factor AP-2 $\beta$ (TFAP2B), via reverse transcription-quantitative polymerase chain reaction in the adipose tissue of the kidney capsule, the mesenterium and subcutaneum, as well as the hypothalamus of obese Zucker diabetic fatty (ZDF) and Zucker lean (ZL) rats aged 22 weeks. All of the target genes, not including SEC16B, exhibited the highest expression levels in the hypothalamus, indicating a critical role of these obesity-associated genes in the central regulation of energy balance (47). Notably, the expression patterns observed in the hypothalamus were not different between the obese ZDF and lean ZL rats (47). However, LYPLAL1, TFAP2B, SEC16B and FAIM2 were expressed significantly less in the kidney fat of the ZDF rats compared with the ZL rats (47). The expression pattern of the investigated obesity genes may imply a role in the central regulation of energy balance, but, for certain genes, an additional role within the adipose tissue (47). However, the role of SEC16B in lipid metabolism in animal models remains to be elucidated.

RGPR-p117, which is a transcription factor $(1,2,8)$, has been shown to enhance the gene expression of regucalcin in kidney cells $(7,8)$. Regucalcin has been found to stimulate adipogenesis in bone marrow mesenchymal stem cells (48), and overexpressed regucalcin was demonstrated to cause diabetes and lipid metabolic disorders with hyperlipidemia in vivo $(49,50)$. RGPR-p117 is speculated to partly participate in lipid metabolic disorder, which is mediated through regucalcin. As RGPR-p117/SEC16B may regulate ER export implicated in lipids (13), RGPR-p117/SEC16B is speculated to contribute to lipid metabolic disorders.

Further studies are required to elucidate the direct evidence regarding the role of RGPR-p117/SEC16B in the regulation of lipid and glucose metabolism, which are implicated in obesity and diabetes. It is possible that the target genes of RGPR-p117 may be involved in human obesity.

\section{Conclusion}

RGPR-p117 was originally discovered as a novel protein that binds to a nuclear factor I consensus motif, TTGGC(N) ${ }_{6} \mathrm{CC}$ in the promoter region of the rgn gene $(1,2)$. Phosphorylated RGPR-p117, which possesses a leucine zipper motif, was demonstrated to be crucial as a transcription factor in the expression of various target genes $(7,9)$. Furthermore, RGPR-p117 localizes in the plasma membranes, mitochondria and ER of modeled kidney cells (34), indicating that this protein has a multifunctional role in the regulation of cellular functions. Notably, RGPR-p117 exhibited a protective role in apoptotic cell death evoked by various stimulatory factors in vitro (37). Additionally, incomplete splicing for the human RGPR-p117 gene is suggested to be involved in carcinogenesis in human placenta $(2,9)$. Thus, RGPR-p117 may have a multifunctional role in cell regulation.

RGPR-p117/SEC16B was proposed to be involved as an ER export factor implicated in the transport of proteins and lipids in the ER (12). As a result of this finding, it was proposed that RGPR-p117 is renamed to SEC16B (12). There is currently growing evidence that RGPR-p117/SEC16B is involved in human obesity and diabetes. However, to the best of our knowledge, the existing studies do not provide direct evidence that RGPR-p117/SEC16B has a regulatory role in lipid metabolism in vivo and in vitro. It is possible that RGPR-p117, a transcription factor, may target the gene expression for molecules associated with lipid metabolism. However, further studies are required to elucidate this fully.

\section{References}

1. Misawa $\mathrm{H}$ and Yamaguchi M: Identification of transcription factor in the promoter region of rat regucalcin gene: Binding of nuclear factor I-A1 to TTGGC motif. J Cell Biochem 84: 795-802, 2000

2. Misawa $\mathrm{H}$ and Yamaguchi M: Molecular cloning and sequencing of the cDNA coding for a novel regucalcin gene promoter region-related protein in rat, mouse and human liver. Int J Mol Med 8: 513-520, 2001.

3. Miasawa $\mathrm{H}$ and Yamaguchi M: Involvement of hepatic nuclear factor I binding motif in transcriptional regulation of $\mathrm{Ca}^{2+}$-binding protein regucalcin gene. Biochem Biophys Res Commun 269: 270-278, 2000.

4. Misawa $\mathrm{H}$ and Yamaguchi $\mathrm{M}$ : Intracellular signaling factors-enhanced hepatic nuclear protein binding to TTGGC sequence in the rat regucalcin gene promoter: Involvement of protein phosphorylation. Biochem Biophys Res Commun 279: 275-281, 2000.

5. Sawada N, Nakagawa T, Murata T and Yamaguchi M: Nuclear localization of a novel protein, RGPR-p117, in cloned normal rat kidney proximal tubular epithelial cells. Int J Mol Med 16: 809-814, 2005.

6. Sawada N and Yamaguchi M: Overexpression of RGPR-p117 enhances regucalcin gene expression in cloned normal rat kidney proximaltubularepithelialcells. IntJMolMed 16: 1049-1055,2005.

7. Sawada N and Yamaguchi M: Overexpression of RGPR-p117 enhances regucalcin gene promoter activity in cloned normal rat kidney proximal tubular epithelial cells: Involvement of TTGGC motif. J Cell Biochem 99: 589-597, 2006.

8. Yamaguchi M: Novel protein RGPR-p117: Its role as the regucalcin gene transcription factor. Mol Cell Biochem 327: 53-63, 2009.

9. Yamaguchi M: Role of regucalcin in calcium signaling. Life Sci 66: 1769-1780, 2000.

10. Yamaguchi M: Role of regucalcin in maintaining cell homeostasis and function (Review). Int J Mol Med 15: 372-389, 2005.

11. Yamaguchi M: Regucalcin and cell regulation: Role as a suppressor in cell signaling. Mol Cell Biochem 353: 101-137, 2011.

12. Bhattacharyya D and Glick BS: Two mammalian Sec16 homologues have nonredundant functions in endoplasmic reticulum (ER) export and transitional ER organization. Mol Biol Cell 18: 839-849, 2007.

13. Budnik A and Stephens DJ: ER exit sites-Localization and control of COPII vesicle formation. FEBS Lett 583: 3796-3803, 2009. 
14. Ng MCY, Tam CHT, So WY, Ho JSK, Chan AW, Lee HM, Wang Y, Lam VKL, Chan JCN and Ma RCW: Implication of genetic variants near NEGR1, SEC16B, TMEM18, ETV5/DGKG, GNPDA2, LIN7C/BDNF, MTCH2, BCDIN3D/FAIM2, SHsB1, FTO, MC4R, and KCTD15 with obesity and type 2 diabetes in 7705 Chinese. J Clin Endocrinol Metab 95: 2418-2425, 2010.

15. Misawa $\mathrm{H}$ and Yamaguchi M: Gene expression for a novel protein RGPR-p117 in various species: The stimulation by intracellular signaling factors. J Cell Biochem 87: 188-193, 2002.

16. Yamaguchi M, Misawa $\mathrm{H}$ and Ma ZJ: Novel protein RGPR-p117: The gene expression in physiologic state and the binding activity to regucalcin gene promoter region in rat liver. J Cell Biochem 88 1092-1100, 2003.

17. Sawada N and Yamaguchi M: A novel regucalcin gene promoter region-related protein: Comparison of nucleotide and amino acid sequences in vertebrate species. Int J Mol Med 15: 97-104, 2005.

18. Bairch A, Bucher P and Hofmann K: The PROSITE database, its status in 1997. Nucleic Acids Res 25: 217-221, 1997.

19. Landschulz WH, Johnson PF and McKnight SL: The leucine zipper: A hypothetical structure common to a new class of DNA binding proteins. Science 240: 1759-1764, 1988.

20. Busch SJ and Sassone-Corsi P: Dimers, leucine zippers and DNA-binding domains. Trends Genet 6: 36-40, 1990.

21. O'Shea EK, Rutkowski R and Kim PS: Evidence that the leucine zipper is a coiled coil. Science 243: 538-542, 1989.

22. Vinson CR, Sigler PB and McKnight SL: Scissors-grip model for DNA recognition by a family of leucine zipper proteins. Science 246: 911-916, 1989.

23. O'Shea EK, Rutkowski R, Stafford WF III and Kim PS Preferential heterodimer formation by isolated leucine zippers from fos and jun. Science 245: 646-648, 1989.

24. Maekawa T, Sakura H, Kanei-Ishii C, Sudo T, Yoshimura T, Fujisawa J, Yoshida $\mathrm{M}$ and Ishii S: Leucine zipper structure of the protein CRE-BP1 binding to the cyclic AMP response element in brain. EMBO J 8: 2023-2028, 1989.

25. Collum RG and Alt FW: Are myc proteins transcription factors? Cancer Cells 2: 69-75, 1990.

26. Clerc RG, Corcoran LM, LeBowitz JH, Baltimore D and Sharp PA: The B-cell-specific Oct-2 protein contains POU boxand homeo box-type domains. Genes Dev 2: 1570-1581, 1988.

27. Verheijen MHG and Defize LHK: Parathyroid hormone activates mitogen-activated protein kinase via a cAMP-mediated pathway independent of rats. J Biol Chem 272: 3423-3429, 1997.

28. Nishizuka Y: Studies and perspectives of protein kinase C. Science 233: 305-312, 1986.

29. Yamaguchi $\mathbf{M}$ and Nakajima M: Involvement of intracellular signaling factors in the serum-enhanced $\mathrm{Ca}^{2+}$-binding protein regucalcin mRNA expression in the cloned rat hepatoma cells (H4-II-E). J Cell Biochem 74: 81-89, 1999.

30. Tamaoki T, Nomoto H, Takahashi I, Kato Y, Morimoto M and Tomita E: Staurosporine, a potent inhibitor of phospholipid/ $\mathrm{Ca}^{2+}$ dependent protein kinase. Biochem Biophys Res Commun 135: 397-402, 1986

31. Peleck SL, Charest DL, Mordret GP, Siow YL, Palaty C, Campbell D, Chaslton L, Samiei M and Sanghera JS: Networking with mitogen-activated protein kinases. Mol Cell Biochem 127: $157-169,1993$.

32. Nakagawa $\mathrm{T}$ and Yamaguchi M: Hormonal regulation on regucalcin mRNA expression in cloned normal rat kidney proximal tubular epithelial NRK52E cells. J Cell Biochem 95: 589-597, 2005.

33. Hunter T: Protein kinases and phosphatases: The Yin and Yang of protein phosphorylation and signaling. Cell 80: 225-236, 1995.

34. Tomono S, Sawada N and Yamaguchi M: Overexpression of RGPR-p117 induces the decrease in protein and DNA contents in cloned normal rat kidney proximal tubular epithelial NRK52E cells. Int J Mol Med 20: 79-83, 2007.
35. Ribeiro JM and Carson DA: $\mathrm{Ca}^{2+} / \mathrm{Mg}^{2+}$-dependent endonuclease from human spleen: Purification, properties, and role in apoptosis. Biochemistry 32: 9129-9136, 1993.

36. Yamaguchi $M$ and Sakurai K: Inhibitory effect of calcium-binding protein regucalcin on $\mathrm{Ca}^{2+}$-activated DNA fragmentation in rat liver nuclei. FEBS Lett 279: 281-284, 2002.

37. Yamaguchi M, Tomono S and Nakagawa T: Overexpression of RGPR-p117 suppresses apoptotic cell death and its related gene expression in cloned normal rat kidney proximal tubular epithelial NRK52E cells. Int J Mol Med 20: 565-571, 2007.

38. Tani K, Tagaya M, Yonekawa S and Baba T: Dual function of Sec16B: Endoplasmic reticulum-derived protein secretion and peroxisome biogenesis in mammalian cells. Cell Logist 1: 164-167, 2011.

39. Yonekawa S, Furuno A, Baba T, Fujiki Y, Ogawasara Y, Yamamoto A, Tagaya M and Tani K: Sec16B is involved in the endoplasmic reticulum export of the peroxisomal membrane biogenesis factor peroxin 16 (Pex16) in mammalian cells. Proc Natl Acad Sci USA 108: 12746-12751, 2011.

40. Budnik A, Heesom KJ and Stephens DJ: Characterization of human Sec16B: Indications of specialized, non-redundant functions. Sci Rep 1: 77, 2011

41. Hotta K, Kitamoto T, Kitamoto A, Mizusawa S, Matsuo T, Nakata Y, Kamohara S, Miyatake N, Kotani K, Komatsu R, et al: Association of variations in the FTO, SCG3 and MTMR9 genes with metabolic syndrome in a Japanese population. J Hum Genet 56: 647-651, 2011.

42. Albuquerque D, Nóbrega C, Rodríguez-López R and Manco L: Association study of common polymorphisms in MSRA, TFAP2B, MC4R, NRXN3, PPARGC1A, TMEM18, SEC16B, HOXB5 and OLFM4 genes with obesity-related traits among Portuguese children. J Hum Genet 59: 307-313, 2014.

43. Williams MJ, Almén MS, Fredriksson R and Schiöth HB: What model organisms and interactomics can reveal about the genetics of human obesity. Cell Mol Life Sci 69: 3819-3834, 2012.

44. Lv D, Zhang DD, Wang H, Zhang Y, Liang L, Fu JF, Xiong F, Liu GL, Gong CX, Luo FH, et al: Genetic variations in SEC16B, MC4R, MAP2K5 and KCTD15 were associated with childhood obesity and interacted with dietary behaviors in Chinese school-age population. Gene 560: 149-155, 2015.

45. Kaewsutthi S, Santiprabhob J, Phonrat B, Tungtrongchitr A, Lertrit $\mathrm{P}$ and Tungtrongchitr R: Exome sequencing in Thai patients with familial obesity. Genet Mol Res 15: 8311, 2016.

46. Ng MC, Tam CH, So WY, Ho JS, Chan AW, Lee HM, Wang Y, Lam VK, Chan JC and Ma RC: Implication of genetic variation near NEGR1, SEC16B, TMEM18, ETV5/DGKG, GNPDA2, LIN7C/BDNF, MTCH2, BCDIN3D/FAIM2, SH2B1, FTO, MC4R and KCTD15 with obesity and type 2 duabetes in 7705 Chinese. J Clin Endocrinol Metab 95: 2418-2425, 2010.

47. Schmid PM, Heid I, Buechler C, Steege A, Resch M, Birner C, Endemann DH, Riegger GA and Luchner A: Expression of fourteen novel obesity-related genes in Zucker diabetic fatty rats. Cardiovas Diabetol 11: 48, 2012.

48. Yamaguchi M, Weitzmann MN, Baile CA and Murata T: Exogenous regucalcin suppresses osteoblastogenesis and stimulates adipogenesis in mouse bone marrow culture. Integr Biol (Camb) 4: 1215-1222, 2012.

49. Yamaguchi M: Regucalcin and metabolic disorders: Osteoporosis and hyperlipidemia are induced in regucalcin transgenic rats. Mol Cell Biochem 341: 119-133, 2010.

50. Yamaguchi $\mathrm{M}$ and Murata T: Involvement of regucalcin in lipid metabolism and diabetes. Metabolism 62: 1045-1051, 2013. 